\title{
Encouraging breastfeeding: The role of fathers
}

\section{> Abstract}

National and international policy and guidance recognise the health and other benefits of breastfeeding. Fathers are often the closest form of social support to the mother, with research suggesting that fathers can be an important source of breastfeeding support; however, they are not acknowledged in many existing breastfeeding protocols or policies. The aim of this literature review is to explore the role of the father in supporting breastfeeding. Nine studies were included in the literature review. Three themes were identified: duration of breastfeeding; educating fathers; and breastfeeding support from fathers. Findings suggest fathers offer a potentially valuable support for breastfeeding mothers. Resources and interventions for fathers need to be designed and researched with a need for policies and guidelines to acknowledge their role.

\section{Key words}

$>$ Breastfeeding > Fathers > Support > Health visitors > Mothers and Ventura, 2009; Scott et al, 2012; McCrory and Layte, 2012), diabetes and cardiovascular disease (Horta and Victora, 2013). Some research suggests that breastfed babies may have better neurological development, and improved performance and intelligence later in life (Horta and Victora, 2013). There is a growing consensus that breastfeeding can offer protection for the mother against the risk of ovarian and breast cancer (World Cancer Research Fund, 2009), as well as promoting breastfeeding, positive parenting and attachment (Gutman et al, 2009; Field, 2010; Wave Trust and Department for Education, 2013).

This evidence base has underpinned both national and international breastfeeding policy with UNICEF, the World Health Organization (WHO), and the US Department of Health and Human Services encouraging breastfeeding from birth (WHO, 2009; US Department of Health and Human Services, 2011; UNICEF, 2013). Likewise, in England, government policy (DHSC, 2009) has recognised breastfeeding as a priority for health professionals. Most recently (DHSC, 2014), it has placed responsibility on health visitors to encourage breastfeeding to positively influence a child's start in life.

Nearly three-quarters of mothers in England initiate breastfeeding (73.8\%) (NHS England, 2015); however, data from Public Health England (PHE) (2016) suggest that only $43.7 \%$ of infants at 6-8 weeks are still breastfed. There are several points in antenatal and postnatal care plans where women should receive information about the benefits of breastfeeding. Often, the midwife initiates discussions about breastfeeding and midwives are identified by mothers as the most common source of information (83\%) on breastfeeding (McAndrew et al, 2012). Once mother and baby leave maternity care, the health visitor becomes the primary professional supporter for continued breastfeeding. However, health visitors do not see the mother and baby until 10-14 days after birth, a crucial period for sustaining breastfeeding. Fathers can support the mother during this time.

Fathers are often the closest form of social support to the mother, with research suggesting 


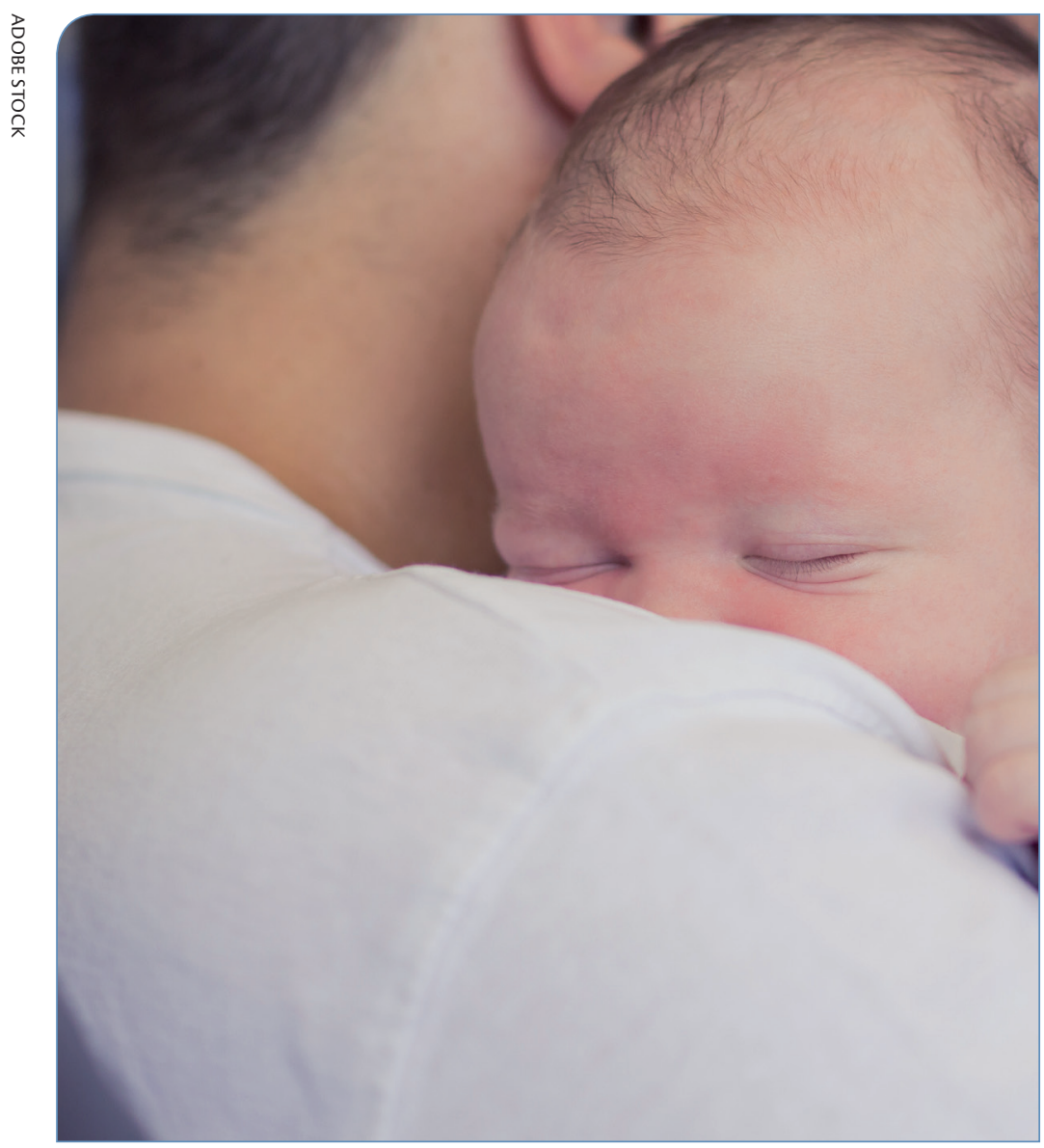

that fathers can be an important source of breastfeeding support (Bar-Yam and Darby, 1997). Despite this, the father's role has not been acknowledged in existing breastfeeding protocols. None of the existing international or UK policy or guidance specifically refer to the role of the father, although some do mention 'partner' support (DHSC, 2009; WHO/UNICEF, 2009; National Institute of Health and Care Excellence (NICE), 2015)

There is currently a gap in the knowledge of fathers' understanding of the value of breastfeeding, their attitude towards it and their interest in supporting their partners. Could they play a more prominent role?

\section{Aims and objectives}

This article reviews the literature exploring the role of fathers in the continuation of partial or exclusive breastfeeding. The methodology was chosen to allow exploration of the existing research, and to critically appraise any strengths and weakness in the published evidence base with a view to examining whether current breastfeeding policies adequately reflect the role of the father.

\section{Methods}

\section{Search strategy}

An initial search was conducted using Google Scholar. This identified the volume of research and helped in finding key terms for the literature search. This process highlighted key studies and focused the project on the period between 2009 and 2016, when most of the research was published. The database EBSCOHost was searched including Academic Search Premier, MEDLINE, Psychology and Behavioural Sciences Collection, PsycINFO, SPORTDiscus and CINAHL Plus with Full Text. Key terms are displayed in Table 1.

At the outset, the following inclusion criteria were identified:

- Publications that originated from western Europe, north America, Canada, Australia or New Zealand (due to similar demographics and cultures to the UK and, therefore, findings more likely to be transferable)

- Primary research

- Studies focusing on fathers' attitudes, knowledge and support in relation to breastfeeding rates or mothers' breastfeeding experiences in the postpartum period

- Studies focusing on fathers (originally, the inclusion criteria were cohabiting biological fathers; however, due to limited findings of this description in studies, the criteria was widened to just fathers)

- Peer-reviewed papers

- Published in English only.

All papers were critically appraised using the Critical Appraisal Skills Programme (CASP) tools, (CASP, 2014). Three different types of the CASP checklist were used: a randomised controlled trial (RCT) checklist; a cohort study checklist; and a qualitative study checklist.

\section{Sample}

The initial search identified 1698 papers. Of these, 1197 were duplicates. The remaining 501 papers were assessed against the inclusion criteria. Nine papers were identified as relevant to this study. Of the nine papers collated for the review, three were conducted in Canada, two in Australia, two in north America, one in the UK and one in Cyprus (Table 2). The studies employed various research designs; three were quantitative studies using an experimental method of a RCT.

Three papers were quantitative studies that used a non-experimental design of a cross-sectional study. Three qualitative studies used a thematic analysis approach. Common trends were identified 
with the quantitative studies through comparison of the findings. The process of deriving themes was completed using a deductive thematic analysis approach. Pre-assigned codes and themes were developed and findings of the papers were used to support them. Following the stages of thematic analysis, themes were identified through coding and categorising data to redefine themes (Braun and Clarke, 2006). Themes and trends identified were:

- Duration of breastfeeding.

- Fathers' education.

- Fathers' support.

\section{Findings}

Duration of breastfeeding

A trend throughout seven studies was the duration of the mother's breastfeeding. Ozluses and Celebioglu (2014) conducted a RCT to explore the exclusive breastfeeding (EBF) rate after mothers and fathers had received EBF education vs mothers only receiving EBF education.

The EBF rate was higher at 6 months for mothers and fathers receiving EBF in comparison to mothers only. The results showed significant differences in the duration of breastfeeding between the groups from the first to the sixth month postpartum. In the study, however, the researcher was not blind to group allocation so there was a risk of contamination between different intervention groups. In comparison to Özlüses and Çelebioglu (2014), Maycock et al (2013) did not find any significant difference in breastfeeding status at 6 months postpartum, in a RCT where fathers received a male-led antenatal education session and 6-week postnatal support package. However, Maycock et al (2013) did find that there was a significant difference in mothers engaging in any breastfeeding at 6 weeks postpartum between the two groups.

In a study by Abbass-Dick et al (2015), although results were non-significant, more mothers were EBF at 6 weeks and 12 weeks postpartum in a RCT intervention group where mothers and fathers received a co-parenting breastfeeding support education package. The study did discover significantly higher rates of any breastfeeding at 12 weeks in the intervention group. There was a high follow-up participation rate in this study, but although the sample was multicultural, participants were middle class, educated and highly motivated to breastfeed. Therefore, the results may not be generalisable to other mothers.

Hunter and Cattelona (2014) conducted a survey to explore breastfeeding initiation and

\begin{tabular}{|c|c|c|c|}
\hline \multirow[b]{2}{*}{$\begin{array}{l}\text { Search database } \\
\text { EBSCOHost Health } \\
\text { Sciences Research } \\
\text { Database } \\
\text { Academic Search Premier, } \\
\text { MEDLINE, Psychology } \\
\text { and Behavioural Sciences } \\
\text { Collection, PsycINFO, } \\
\text { SPORTDiscus and } \\
\text { CINAHLPlus with Full Text }\end{array}$} & \multicolumn{3}{|l|}{ Search terms } \\
\hline & Breastfeeding & $\begin{array}{l}\text { AND } \\
\text { Assistance } \\
\text { Attitude } \\
\text { Encourage } \\
\text { Help } \\
\text { Involvement } \\
\text { Knowledge } \\
\text { Role } \\
\text { Support }\end{array}$ & $\begin{array}{l}\text { AND } \\
\text { Dad } \\
\text { Father } \\
\text { Husband } \\
\text { Men } \\
\text { Partner } \\
\text { Paternal }\end{array}$ \\
\hline
\end{tabular}

duration, and partner support in the first 48 hours of the postpartum period. Findings revealed that EBF at 6 months was significantly higher among mothers who reported involvement and support from their partners. The study showed a big effect, albeit reliant on subjective data of mothers' self-reporting. Mothers were recruited via two different types of antenatal classes, but no difference in effect on breastfeeding experience was found between classes. The study did, however, concentrate on first-time mothers limiting any bias for previous breastfeeding experience. There were also no strict inclusion criteria, suggesting that findings could be generalisable to other breastfeeding mothers.

Maternal confidence and maternal satisfaction were identified as themes in three studies. Mannion et al (2013) conducted a survey to identify mothers' perceived partner support and breastfeeding self-efficacy, finding that partner support was associated with higher maternal confidence in breastfeeding. Women who perceived positive or active support from their partner had higher self-efficacy and breastfed longer in comparison to those who did not report positive partner support.

Mannion et al (2013) also found no significant difference between those mothers who perceived positive support and those who did not in their perception of milk production. It concluded that partner support has a greater influence on breastfeeding confidence than the mother's perception of her milk production. The study had self-selection bias, where participants chose

\section{"There is a gap in the knowledge of fathers' understanding of the value of breastfeeding, their attitude towards it and their interest in supporting their partners «}




\begin{tabular}{|c|c|c|c|c|c|c|}
\hline $\begin{array}{l}\text { Journal } \\
\text { article }\end{array}$ & Study aims & $\begin{array}{l}\text { Sample } \\
\text { characteristics }\end{array}$ & Data collection & Data analysis & Findings & $\begin{array}{l}\text { Study } \\
\text { limitations }\end{array}$ \\
\hline $\begin{array}{l}\text { Tohotoa, J, } \\
\text { Maycock, } \\
\text { B, Hauck, } \\
\text { YL, Howat } \\
\text { P, Burns S, } \\
\text { Binns CW } \\
\text { Dads make } \\
\text { a difference: } \\
\text { An } \\
\text { exploratory } \\
\text { study of } \\
\text { paternal } \\
\text { support for } \\
\text { breastfeeding } \\
\text { in Perth, } \\
\text { Western } \\
\text { Australia. } \\
\text { International } \\
\text { Breastfeeding } \\
\text { Journal } \\
\text { Australia, } \\
\text { 2009 }\end{array}$ & $\begin{array}{l}\text { To identify } \\
\text { what women } \\
\text { and men } \\
\text { perceive as } \\
\text { essential } \\
\text { paternal } \\
\text { support to } \\
\text { facilitate } \\
\text { successful } \\
\text { breastfeeding. } \\
\text { What men } \\
\text { believe they } \\
\text { need to assist } \\
\text { them to be } \\
\text { a successful } \\
\text { breastfeeding } \\
\text { advocate }\end{array}$ & $\begin{array}{l}48 \text { postnatal } \\
\text { mothers. } \\
\text { Breastfeeding } \\
\text { or had in past } 6 \\
\text { months. } 28 \text { men. } \\
\text { Recruited via } \\
\text { posters at child } \\
\text { health clinics, } \\
\text { child care centres } \\
\text { and early learning } \\
\text { centres. Fathers } \\
\text { recruited by their } \\
\text { partners with } \\
\text { 'wanted' notices at } \\
\text { the above centres. } \\
\text { Mainly first-time } \\
\text { parents, married or } \\
\text { living together }\end{array}$ & $\begin{array}{l}8 \text { focus groups of } \\
4-10 \text { mothers each } \\
\text { using interview } \\
\text { questions. } \\
\text { Fathers variety of } \\
\text { means, telephone } \\
\text { interviews, online } \\
\text { survey and focus } \\
\text { groups }\end{array}$ & $\begin{array}{l}\text { Qualitative } \\
\text { data thematic } \\
\text { analysis. Coding } \\
\text { and categorising } \\
\text { themes. Two } \\
\text { major themes: } \\
\text { 'Dads make a } \\
\text { difference' and } \\
\text { 'Wanting to be } \\
\text { involved'. Mothers } \\
\text { subthemes: } \\
\text { 'Anticipating } \\
\text { needs and getting } \\
\text { the job done', } \\
\text { 'Encouraging to } \\
\text { do your best' } \\
\text { and 'Paternal } \\
\text { commitment to } \\
\text { breastfeeding'. } \\
\text { Fathers } \\
\text { subthemes: } \\
\text { 'Wanting relevant } \\
\text { information', } \\
\text { 'Learning the role' } \\
\text { and 'Being an } \\
\text { advocate' }\end{array}$ & $\begin{array}{l}\text { Fathers' support } \\
\text { essential to the } \\
\text { mother being } \\
\text { able to breastfeed } \\
\text { successfully. } \\
\text { Mothers identified } \\
\text { that fathers should } \\
\text { encourage and } \\
\text { problem solve. } \\
\text { Fathers identified } \\
\text { that they require } \\
\text { information, } \\
\text { recognition of their } \\
\text { role and involvement } \\
\text { in antenatal classes } \\
\text { for them to be } \\
\text { advocates and } \\
\text { supporters } \\
\text { of breastfeeding }\end{array}$ & $\begin{array}{l}\text { Non- } \\
\text { representative } \\
\text { self-selected } \\
\text { sample. } \\
\text { Small sample }\end{array}$ \\
\hline $\begin{array}{l}\text { Rempel LA, } \\
\text { Rempel JK, } \\
\text { Moore KCJ. } \\
\text { Relationships } \\
\text { between } \\
\text { types of } \\
\text { father } \\
\text { breastfeeding } \\
\text { support and } \\
\text { breastfeeding } \\
\text { outcomes. } \\
\text { Canada, 2017 } \\
\text { Maternal } \\
\text { and Child } \\
\text { Nutrition }\end{array}$ & $\begin{array}{l}\text { To examine } \\
\text { the } \\
\text { relationship } \\
\text { between } \\
\text { fathers' } \\
\text { reported and } \\
\text { mothers' } \\
\text { perceptions of } \\
\text { breastfeeding } \\
\text { support and } \\
\text { mothers' } \\
\text { breastfeeding } \\
\text { intentions, } \\
\text { satisfaction } \\
\text { and duration }\end{array}$ & $\begin{array}{l}\text { Study 1: } 64 \text { women } \\
\text { and } 41 \text { men ( } 34 \\
\text { couples) with } \\
\text { children born in } \\
\text { past year. Sample } \\
\text { identified from } \\
\text { newspaper birth } \\
\text { announcements } \\
\text { and recruited by } \\
\text { telephone. } \\
\text { Study 2: } 80 \text { mothers } \\
\text { and } 63 \text { fathers } \\
\text { had originally } \\
\text { participated in } \\
\text { breastfeeding best } \\
\text { practice guidelines } \\
\text { implementation } \\
\text { study (Rempel and } \\
\text { McCleary, 2012), } \\
\text { initially recruited } \\
\text { from hospitals }\end{array}$ & $\begin{array}{l}\text { Questionnaires } \\
\text { either online or } \\
\text { by mail. Partner } \\
\text { breastfeeding } \\
\text { influence scale } \\
\text { (PBIS) used to } \\
\text { measure mothers' } \\
\text { and fathers' } \\
\text { breastfeeding } \\
\text { duration intention. } \\
\text { Breastfeeding } \\
\text { experiences rated } \\
\text { by mothers and } \\
\text { fathers using } \\
\text { breastfeeding } \\
\text { satisfaction } \\
\text { scale. Study } \\
\text { 2: } 12 \text { months' } \\
\text { post-partum } \\
\text { follow-up study. } \\
\text { Questionnaire by } \\
\text { telephone or mail. } \\
\text { PBIS, maternal } \\
\text { breastfeeding } \\
\text { evaluation scale, } \\
\text { breastfeeding } \\
\text { status and duration } \\
\text { of breastfeeding }\end{array}$ & Statistical analysis & $\begin{array}{l}\text { Responsiveness most } \\
\text { frequent influence } \\
\text { behaviour. Mothers' } \\
\text { breastfeeding } \\
\text { satisfaction and } \\
\text { success rates } \\
\text { significantly positively } \\
\text { correlated with } \\
\text { her perceptions } \\
\text { of partner's } \\
\text { responsiveness during } \\
\text { breastfeeding. When } \\
\text { fathers claimed } \\
\text { breastfeeding } \\
\text { support behaviours of } \\
\text { appreciation, presence } \\
\text { and informed about } \\
\text { breastfeeding, mothers } \\
\text { actually breastfed for } \\
\text { shorter time. May not } \\
\text { have been sensitive } \\
\text { and responsive } \\
\text { enough if mother } \\
\text { having difficulties and } \\
\text { affected self-efficacy } \\
\text { and autonomy }\end{array}$ & $\begin{array}{l}\text { Small } \\
\text { sample sizes. } \\
\text { Sample not } \\
\text { representative } \\
\text { of wider } \\
\text { population. } \\
\text { Breastfeeding } \\
\text { behaviours } \\
\text { may not be } \\
\text { representative } \\
\text { of different } \\
\text { cultures }\end{array}$ \\
\hline
\end{tabular}




\begin{tabular}{|c|c|c|c|c|c|}
\hline $\begin{array}{l}\text { Journal } \\
\text { article }\end{array}$ & Study aims & $\begin{array}{l}\text { Sample } \\
\text { characteristics }\end{array}$ & Data collection & Findings & $\begin{array}{l}\text { Study } \\
\text { limitations }\end{array}$ \\
\hline $\begin{array}{l}\text { Abbass-Dick } \\
\text { J, Stern SB, } \\
\text { Nelson LE, } \\
\text { Watson W, } \\
\text { Dennis C. } \\
\text { Coparenting } \\
\text { Breastfeeding } \\
\text { support and } \\
\text { exclusive } \\
\text { breastfeeding: } \\
\text { a randomised } \\
\text { control trial. } \\
\text { Pediatrics } \\
\text { Canada, } 2015\end{array}$ & $\begin{array}{l}\text { To evaluate } \\
\text { the effect of a } \\
\text { co-parenting } \\
\text { breastfeeding } \\
\text { support } \\
\text { intervention } \\
\text { on exclusive } \\
\text { breastfeeding }\end{array}$ & $\begin{array}{l}\text { Primiparas } \\
\text { mothers } \\
\text { and fathers } \\
\text { cohabiting. } \\
\text { Recruited from } \\
\text { hospital in the } \\
\text { first } 2 \text { days } \\
\text { postpartum. } \\
107 \text { couples in } \\
\text { intervention } \\
\text { group }\end{array}$ & $\begin{array}{l}\text { Follow-up data collected at } \\
6 \text { weeks postpartum from } \\
\text { mothers and fathers, and from } \\
\text { mothers alone at } 12 \text { weeks, } \\
\text { which included breastfeeding } \\
\text { status, duration and how } \\
\text { much interventions learnt in } \\
\text { the group were used. } 12 \text { weeks } \\
\text { co-parenting relationship scale. } \\
\text { Brief scale used at baseline and } \\
6 \text { weeks. Postpartum partner } \\
\text { support scale at } 6 \text { weeks. } \\
\text { Breastfeeding self-efficacy scale } \\
\text { adapted to assess paternal } \\
\text { breastfeeding self-efficacy } \\
\text { to measure confidence in } \\
\text { assisting the mother. Baseline } \\
\text { and } 6 \text { weeks. Paternal infant } \\
\text { feeding attitude scale at } \\
\text { baseline and } 6 \text { weeks for } \\
\text { fathers. Mothers breastfeeding } \\
\text { support } 6 \text { and } 12 \text { week who } \\
\text { supported and frequency. } \\
\text { Self-report online or telephone } \\
\text { questionnaire }\end{array}$ & $\begin{array}{l}\text { Co-parenting } \\
\text { intervention increased } \\
\text { breastfeeding duration } \\
\text { at } 12 \text { weeks significantly. } \\
\text { Duration rate at } 6 \\
\text { weeks and exclusivity } \\
\text { rates at } 6 \text { and } 12 \\
\text { weeks higher than } \\
\text { control group but not } \\
\text { significant. Significant } \\
\text { improvement in } \\
\text { paternal breastfeeding } \\
\text { self-efficacy over first } 6 \\
\text { weeks in intervention } \\
\text { group. Significantly } \\
\text { more mothers received } \\
\text { breastfeeding help from } \\
\text { fathers in first } 6 \text { weeks } \\
\text { and were satisfied with } \\
\text { father involvement and } \\
\text { information received }\end{array}$ & $\begin{array}{l}\text { Possibly } \\
\text { measured too } \\
\text { early to detect } \\
\text { significant } \\
\text { difference } \\
\text { in exclusive } \\
\text { breastfeeding } \\
\text { between the } \\
\text { groups. Highly } \\
\text { motivated to } \\
\text { breastfeeding } \\
\text { sample. Limited } \\
\text { variability. } \\
\text { Selection bias. } \\
\text { Eligibility. } \\
\text { Postpartum } \\
\text { period busy } \\
\text { time to review } \\
\text { information } \\
\text { could have } \\
\text { been delivered } \\
\text { prenatally }\end{array}$ \\
\hline $\begin{array}{l}\text { Hunter T, } \\
\text { Cattelona G. } \\
\text { Breastfeeding } \\
\text { initiation } \\
\text { and duration } \\
\text { in first time } \\
\text { mothers: } \\
\text { Exploring } \\
\text { the impact } \\
\text { of father } \\
\text { involvement } \\
\text { in the early } \\
\text { postpartum } \\
\text { period. } \\
\text { USA, 2014. } \\
\text { Health } \\
\text { Promotion } \\
\text { Perspectives }\end{array}$ & $\begin{array}{l}\text { To explore the } \\
\text { influence of social } \\
\text { support of the } \\
\text { male partner on } \\
\text { first-time mothers' } \\
\text { breastfeeding } \\
\text { initiation and } \\
\text { duration. To assess } \\
\text { the level of father } \\
\text { involvement and } \\
\text { support. Assess } \\
\text { the partner } \\
\text { participation in } \\
\text { breastfeeding } \\
\text { decisions }\end{array}$ & $\begin{array}{l}\text { First-time } \\
\text { mothers } \\
\text { recruited from } \\
\text { Bloomington } \\
\text { Area Birth } \\
\text { Services, Indiana. } \\
\text { Had a baby in } \\
\text { last } 12 \text { months. } \\
146 \text { mothers }\end{array}$ & $\begin{array}{l}\text { Self-administered questionnaire } \\
\text { assessing demographics, } \\
\text { breastfeeding initiation, early } \\
\text { post-partum breastfeeding, } \\
\text { breastfeeding plans, support } \\
\text { and duration }\end{array}$ & $\begin{array}{l}\text { Prevalence of exclusive } \\
\text { breastfeeding at } 6 \\
\text { months significantly } \\
\text { higher among mothers } \\
\text { who had involvement } \\
\text { and support from fathers } \\
\text { during the early post- } \\
\text { partum period (first } 48 \\
\text { hours) }\end{array}$ & \\
\hline
\end{tabular}

to take part in the study, which affected the generalisability of the results. The study also relied on subjective self-report data from mothers' perceptions, which could increase the risk of recall bias.

Tohotoa et al (2009) found that mothers felt increased self-confidence in relation to her partner's support. This study conducted eight focus groups to explore mothers' perceptions of her partner support and to ascertain partners' perceptions of the help they needed to support the mother effectively. The study had limited success in recruiting fathers, although used a range of data collection methods to overcome this limitation. Fathers were recruited via mothers, suggesting a possible sample of the most committed mothers to breastfeeding risking self-selection bias.

In a quantitative study, Rempel et al (2017) found maternal satisfaction in breastfeeding to be a protective factor in reducing the risk of early 


\begin{tabular}{|c|c|c|c|c|c|c|}
\hline $\begin{array}{l}\text { Journal } \\
\text { article }\end{array}$ & Study aims & $\begin{array}{l}\text { Sample } \\
\text { characteristics }\end{array}$ & Data collection & $\begin{array}{l}\text { Data } \\
\text { analysis }\end{array}$ & Findings & Study limitations \\
\hline $\begin{array}{l}\text { Mannion CA, } \\
\text { Hobbs AJ, } \\
\text { McDonald } \\
\text { SW, Tough SC. } \\
\text { Maternal } \\
\text { Perceptions } \\
\text { of partner } \\
\text { support } \\
\text { during } \\
\text { breastfeeding. } \\
\text { International } \\
\text { Breastfeeding } \\
\text { Journal } \\
\text { Canada, } 2013\end{array}$ & $\begin{array}{l}\text { To find out } \\
\text { if mothers } \\
\text { reporting } \\
\text { positive } \\
\text { support from } \\
\text { their partners } \\
\text { would have } \\
\text { a higher } \\
\text { confidence } \\
\text { in breast milk } \\
\text { production } \\
\text { and higher } \\
\text { breastfeeding } \\
\text { self-efficacy }\end{array}$ & $\begin{array}{l}\text { Convenience } \\
\text { sample from } \\
\text { a larger study. } \\
\text { Recruitment } \\
\text { posters at } \\
\text { health centres, } \\
\text { breastfeeding } \\
\text { women } \\
\text { approached } \\
\text { and referred } \\
\text { to research } \\
\text { assistants. } \\
76 \text { mothers. } \\
\text { Inclusion } \\
\text { currently } \\
\text { or recently } \\
\text { breastfeeding } \\
\text { women currently } \\
\text { with the partner }\end{array}$ & $\begin{array}{l}\text { Breastfeeding } \\
\text { self-efficacy scale } \\
\text { and the Hill and } \\
\text { Humenick Lactation } \\
\text { scale perceived milk } \\
\text { supply. Demographic } \\
\text { questionnaire } \\
\text { also addressed } \\
\text { breastfeeding } \\
\text { status, duration and } \\
\text { variables that can } \\
\text { affect breastfeeding. } \\
\text { Also perceived } \\
\text { partner support }\end{array}$ & $\begin{array}{l}\text { Statistical } \\
\text { analysis. } \\
\text { Responses } \\
\text { to questions } \\
\text { of perceived } \\
\text { partner } \\
\text { support } \\
\text { categorised } \\
\text { into } \\
\text { positive, } \\
\text { negative/ } \\
\text { ambivalent }\end{array}$ & $\begin{array}{l}\text { Women who } \\
\text { perceived active or } \\
\text { positive support } \\
\text { from their partner } \\
\text { in breastfeeding had } \\
\text { higher self-efficacy } \\
\text { scores than those who } \\
\text { perceived negative or } \\
\text { ambivalent support. } \\
\text { Only } 5 \% \text { discussed } \\
\text { decision to breastfeed } \\
\text { with partner. No } \\
\text { differences in } \\
\text { perceived support } \\
\text { and lactation scale }\end{array}$ & $\begin{array}{l}\text { Non-representative } \\
\text { sample of wider } \\
\text { population. Majority } \\
\text { highly educated and } \\
\text { only majority living } \\
\text { with partner. Only } \\
\text { focuses on maternal } \\
\text { perceptions, could } \\
\text { have changed over } \\
\text { time. Self-report- } \\
\text { recall bias, could } \\
\text { have forgotten initial } \\
\text { perceptions. } \\
\text { Convenience sample- } \\
\text { selection bias. Small } \\
\text { sample size limits } \\
\text { generalisability }\end{array}$ \\
\hline $\begin{array}{l}\text { Maycock B, } \\
\text { Binns CW, } \\
\text { Dhaliwal } \\
\text { S, Tohotoa } \\
\text { J, Hauck } \\
\text { Y, Burns S, } \\
\text { Howat P. } \\
\text { Education } \\
\text { and Support } \\
\text { for fathers } \\
\text { improves } \\
\text { breastfeeding } \\
\text { rates: A } \\
\text { randomised } \\
\text { control trial. } \\
\text { Journal of } \\
\text { Human } \\
\text { Lactation } \\
\text { Australia, } 2013\end{array}$ & $\begin{array}{l}\text { To investigate } \\
\text { the effects of } \\
\text { an antenatal } \\
\text { education } \\
\text { package and } \\
\text { postnatal } \\
\text { support } \\
\text { targeted to } \\
\text { fathers on the } \\
\text { initiation and } \\
\text { duration of } \\
\text { breastfeeding }\end{array}$ & $\begin{array}{l}\text { Sample recruited } \\
\text { from eight } \\
\text { maternity } \\
\text { hospitals. Fathers } \\
\text { had to intend } \\
\text { to participate in } \\
\text { the rearing of the } \\
\text { child. } 295 \text { men } \\
\text { attended session } \\
\text { and responded } \\
\text { to 6-week } \\
\text { questionnaire }\end{array}$ & $\begin{array}{l}\text { Questionnaires } \\
\text { antenatal and } \\
\text { postnatal } 6 \text { weeks } \\
\text { and } 6 \text { months. } \\
\text { Breastfeeding status } \\
\text { assessed at } 6 \text { weeks. } \\
\text { Fathers followed up } \\
\text { by questionnaire } \\
\text { or by telephone. } \\
\text { lowa Infant Feeding } \\
\text { Attitude Scale } \\
\text { measured at baseline } \\
\text { and } 6 \text { months }\end{array}$ & $\begin{array}{l}\text { Statistical } \\
\text { analysis }\end{array}$ & $\begin{array}{l}\text { Any breastfeeding } \\
\text { rates at } 6 \text { weeks } \\
\text { significantly greater } \\
\text { in intervention group } \\
\text { and less formula use. } \\
\text { Exclusivity at } 6 \text { weeks } \\
\text { non-significant. No } \\
\text { significant differences } \\
\text { at } 6 \text { months between } \\
\text { the groups }\end{array}$ & $\begin{array}{l}\text { Breastfeeding } \\
\text { rates higher in } \\
\text { Australia than other } \\
\text { countries, harder } \\
\text { to measure change. } \\
\text { Small chance of } \\
\text { contamination } \\
\text { between control } \\
\text { and intervention } \\
\text { group. Did not } \\
\text { account for previous } \\
\text { breastfeeding } \\
\text { experience. } \\
\text { Intervention } \\
\text { stopped at } 6 \text { weeks, } \\
\text { contributes to } \\
\text { lack of significant } \\
\text { difference at } 6 \\
\text { months }\end{array}$ \\
\hline $\begin{array}{l}\text { Nickerson } \\
\text { LE, Sykes } \\
\text { AC, Fung } \\
\text { TT. Mothers } \\
\text { Experience } \\
\text { of fathers' } \\
\text { support for } \\
\text { breastfeeding. } \\
\text { Public Health } \\
\text { Nutrition } \\
\text { USA, } 2012\end{array}$ & $\begin{array}{l}\text { To examine } \\
\text { mothers' } \\
\text { experiences } \\
\text { of support } \\
\text { received from } \\
\text { fathers for } \\
\text { breastfeeding }\end{array}$ & $\begin{array}{l}\text { Convenience } \\
\text { sample. Living } \\
\text { with biological } \\
\text { father. Had } \\
\text { baby in last } 12 \\
\text { months. No more } \\
\text { than } 3 \text { months } \\
\text { since stopped } \\
\text { breastfeeding } \\
\text { and breastfed for } \\
\text { at least a month }\end{array}$ & $\begin{array}{l}19 \text { semi-structured } \\
\text { interviews using } \\
\text { questions on type } \\
\text { of support received } \\
\text { from fathers for } \\
\text { breastfeeding. } \\
\text { Questions included } \\
\text { mother's opinion of } \\
\text { the role of the father } \\
\text { in breastfeeding } \\
\text { process, preparation } \\
\text { of the father for } \\
\text { breastfeeding and } \\
\text { what is absent in the } \\
\text { fathers support for } \\
\text { breastfeeding }\end{array}$ & $\begin{array}{l}\text { Thematic } \\
\text { analysis. } \\
\text { Ten } \\
\text { thematic } \\
\text { categories } \\
\text { found }\end{array}$ & $\begin{array}{l}\text { Mothers perceived } \\
\text { fathers to play an } \\
\text { important role in } \\
\text { breastfeeding process } \\
\text { by emotionally and } \\
\text { physically supporting } \\
\text { the mother and are } \\
\text { a critical component } \\
\text { to success. Fathers' } \\
\text { support was crucial } \\
\text { during breastfeeding } \\
\text { challenges to help } \\
\text { them persevere and } \\
\text { continue }\end{array}$ & $\begin{array}{l}\text { Not representative } \\
\text { of populations in } \\
\text { other areas of the } \\
\text { USA. } \\
\text { Socially and } \\
\text { economically } \\
\text { homogenous } \\
\text { sample. Mothers' } \\
\text { perception only }\end{array}$ \\
\hline
\end{tabular}




\begin{tabular}{|c|c|c|c|c|c|c|}
\hline $\begin{array}{l}\text { Journal } \\
\text { article }\end{array}$ & Study aims & $\begin{array}{l}\text { Sample } \\
\text { characteristics }\end{array}$ & Data collection & $\begin{array}{l}\text { Data } \\
\text { analysis }\end{array}$ & Findings & $\begin{array}{l}\text { Study } \\
\text { limitations }\end{array}$ \\
\hline $\begin{array}{l}\text { Datta J, } \\
\text { Graham B, } \\
\text { Wellings K. } \\
\text { The role of } \\
\text { fathers in } \\
\text { breastfeeding: } \\
\text { Decision } \\
\text { making and } \\
\text { support. } \\
\text { British Journal } \\
\text { of Midwifery. } \\
\text { UK, } 2012\end{array}$ & $\begin{array}{l}\text { To evaluate an } \\
\text { intervention } \\
\text { to provide } \\
\text { fathers } \\
\text { information } \\
\text { about } \\
\text { breastfeeding. } \\
\text { Women } \\
\text { targeted to } \\
\text { gather views } \\
\text { on the role } \\
\text { of fathers in } \\
\text { relation to } \\
\text { breastfeeding }\end{array}$ & $\begin{array}{l}14 \text { men and } \\
4 \text { women. } \\
\text { Recruited at the } \\
\text { hospital when } \\
\text { attending for } \\
20 \text {-week scan } \\
\text { or following } \\
\text { delivery on } \\
\text { postnatal ward- } \\
\text { took advice from } \\
\text { midwifes as to } \\
\text { who to approach. } \\
\text { Recruitment via } \\
\text { the mothers in } \\
\text { some instances. } \\
\text { Inclusion- } \\
\text { breastfeeding } \\
\text { mother or } \\
\text { planning to } \\
\text { breastfeed }\end{array}$ & $\begin{array}{l}\text { Interviews questions } \\
\text { on general views } \\
\text { on breastfeeding, } \\
\text { decision making } \\
\text { regarding infant } \\
\text { feeding, father's role } \\
\text { in breastfeeding } \\
\text { and experiences of } \\
\text { breastfeeding }\end{array}$ & $\begin{array}{l}\text { Thematic } \\
\text { analysis }\end{array}$ & $\begin{array}{l}\text { Fathers felt it was mother's } \\
\text { decision to breastfeed, } \\
\text { although saw it as their } \\
\text { role to provide support. } \\
\text { Implication is that some } \\
\text { women may not start } \\
\text { breastfeeding without } \\
\text { partner support in } \\
\text { decision making. Father's } \\
\text { role in providing practical } \\
\text { and emotional support } \\
\text { may be essential to } \\
\text { breastfeeding success. } \\
\text { Fathers providing } \\
\text { encouragement, support } \\
\text { enabled mothers to } \\
\text { continue breastfeeding } \\
\text { despite facing difficulties. } \\
\text { Information pack } \\
\text { provided new knowledge } \\
\text { and strengthened existing } \\
\text { attitude of breastfeeding }\end{array}$ & $\begin{array}{l}\text { Initiation rates } \\
\text { already high in } \\
\text { the area of UK. } \\
\text { Findings may not } \\
\text { be generalised to } \\
\text { other areas of UK. } \\
\text { The sample was } \\
\text { positive about } \\
\text { breastfeeding- } \\
\text { not generalisable- } \\
\text { selection bias. } \\
\text { Small sample size. } \\
\text { Well educated } \\
\text { participants. } \\
\text { Some couples } \\
\text { changed their } \\
\text { mind about } \\
\text { participating } \\
\text { due to having } \\
\text { difficulties } \\
\text { breastfeeding }\end{array}$ \\
\hline $\begin{array}{l}\text { Ozluses E, } \\
\text { Celebioglu } \\
\text { A. Educating } \\
\text { fathers to } \\
\text { improve } \\
\text { breastfeeding } \\
\text { rates and } \\
\text { paternal infant } \\
\text { attachment. } \\
\text { Indian } \\
\text { Pediatrics. } \\
\text { Turkey, } 2014\end{array}$ & $\begin{array}{l}\text { To determine } \\
\text { the effect of } \\
\text { breastfeeding } \\
\text { education } \\
\text { provided } \\
\text { to fathers } \\
\text { on the rate } \\
\text { of EBF and } \\
\text { paternal infant } \\
\text { attachment }\end{array}$ & $\begin{array}{l}\text { Parents of infants } \\
\text { younger than } \\
6 \text { months. } 117 \\
\text { families }\end{array}$ & $\begin{array}{l}\text { Infant feeding type } \\
\text { for EBF rate checked } \\
\text { at } 1,2,4,6 \text { months. } \\
\text { Using interviews } \\
\text { during check-ups. } \\
\text { Paternal infant } \\
\text { attachment scale } \\
\text { used to measure } \\
\text { strength of the bond } \\
\text { between father and } \\
\text { infant }\end{array}$ & $\begin{array}{l}\text { Statistical } \\
\text { analysis }\end{array}$ & $\begin{array}{l}\text { From 1- } 6 \text { months } \\
\text { significant differences } \\
\text { between groups. EBF rates } \\
\text { high at } 6 \text { months when } \\
\text { mother and father both } \\
\text { educated. Paternal infant } \\
\text { attachment score higher } \\
\text { in group with fathers } \\
\text { educated also }\end{array}$ & \\
\hline
\end{tabular}

cessation of breastfeeding. This work presented the results of two surveys, which explored how when fathers engaged in breastfeeding this influenced behaviours. The effect on the breastfeeding intention, satisfaction of the experience and the duration of breastfeeding was explored.

To measure how often fathers engaged in breastfeeding behaviours, a partner breastfeeding influence scale (PBIS) was used, which included subscales of being 'breastfeeding savvy', helping, appreciation, breastfeeding presence and responsiveness. Mothers who reported perceptions of their partner's presence and responsiveness during the breastfeeding experience was positively correlated with greater breastfeeding satisfaction.

Partner responsiveness was also associated with a longer duration of breastfeeding (Rempel et al, 2017). It is questionable as to whether the PBIS is a validated tool, and the PBIS used a Likert scale, which is subjective and open to different participant interpretations. Each study used different samples, although both were highly educated, affluent and not culturally diverse. The recruitment processes have selection bias, which opened the generalisability of the results to question. The first study recruited via birth announcements in the local newspaper and the second study used a small convenience sample; mothers chose to take part, recruited through public health nurses.

\section{Educating fathers}

A theme that emerged through the thematic analysis was fathers' education of breastfeeding, with codes of information, education and knowledge identified in several studies. 


\section{" More mothers reported receiving help from their partner and being satisfied with the partner's involvement when fathers received the co-parenting intervention «}

\author{
Fathers receiving education manuals on \\ breastfeeding and an education session on \\ the technical processes of breastfeeding were \\ suggested by Özlüses and Çelebioglu (2014) \\ to have influenced the mother's duration of \\ breastfeeding. Similarly, Maycock et al (2013) \\ concluded that an education session for fathers \\ promoted breastfeeding and influenced mothers' \\ breastfeeding rates. The education session and \\ additional 6-week follow-up package was aimed \\ to increase fathers' knowledge, skills and problem- \\ solving abilities in infant feeding by educating \\ fathers about the benefits of breastfeeding, \\ difficulties that can be encountered and how to \\ support the breastfeeding mother (Maycock et \\ al, 2013).
}

Maycock et al (2013) indicated that fathers attending an education session in the intervention group had an influence on the mother's attitude and knowledge towards breastfeeding, with higher maternal favourable attitudes to breastfeeding compared to the control group.

In a study by Abbas-Dick et al (2015), fathers were given breastfeeding information on the postnatal ward and a workbook, video and website to access at home. They ascertained that fathers participating in the programme had high paternal self-efficacy and infant feeding attitudes compared to the control group (Abbas-Dick et al, 2015). More mothers reported receiving help from their partner and being satisfied with the partner's involvement when fathers received the co-parenting intervention (Abbas-Dick et al, 2015).

Two studies examined fathers attending antenatal breastfeeding classes, which was collapsed into the education code (Datta et al, 2012; Nickerson et al, 2012). Nickerson et al (2012) interviewed mothers to explore the mother's breastfeeding experience and the support she received from the father. Nickerson et al (2012) indicated that mothers reported feeling that their husbands benefited from attending antenatal breastfeeding classes and spending time with a lactation consultant as they could remember the information and apply the knowledge to support the mother during breastfeeding difficulties. However, a small sample size of non-culturally diverse, educated mothers from an urban area, meant that results may not be generalisable to the whole area. Although the study only looked at mothers' perceptions of support, it did account for recall bias by ensuring that mothers had stopped breastfeeding in the last 3 months.

Datta et al (2012) interviewed mothers and fathers to evaluate a breastfeeding information pack for fathers. They also looked at the father's role in decision making regarding breastfeeding and fathers' experiences of breastfeeding challenges faced in supporting the mother. Datta et al's (2012) results highlighted that fathers had attended antenatal classes and read information on breastfeeding, but few were prepared for problems arising during breastfeeding. Only one father in the study reported that the antenatal class had alerted him to possible difficulties (Datta et al, 2012). However, Datta et al (2012) acknowledged limitations in the small sample size of parents who were educated, older and positive about breastfeeding, creating a self-selection bias. The study area also had higher breastfeeding rates in comparison to the rest of the UK. Datta et al (2012) emphasised that their findings should be interpreted with caution.

Breastfeeding knowledge was a code identified through the thematic analysis, with four studies highlighting that fathers supported the mother's decision to breastfeed as they were aware of the health benefits of breastfeeding (Tohotoa et al, 2009; Datta et al, 2012; Nickerson et al, 2012; Mannion et al, 2013). Despite this, Datta et al (2012) found that some fathers still lacked knowledge of specific benefits to breastfeeding and generally felt it was the mother's decision to breastfeed and whether to continue during the challenges of breastfeeding.

Fathers reported feeling they were able to encourage the mother to continue breastfeeding by providing information on the benefits (Datta et al, 2012; Nickerson et al, 2012). This supported Tohotoa et al's (2009) findings that mothers reported fathers to be committed and supportive towards breastfeeding as they were knowledgeable about the benefits. Mothers also reported fathers being able to help reduce breastfeeding positioning and attachment difficulties for them by researching on the internet (Tohotoa et al, 2009). Some fathers felt inadequate in their knowledge and ability to effectively support the mother and felt unprepared for the fathering role (Tohotoa et al, 2009). Fathers expressed wanting more information on difficulties in breastfeeding and how they could support the mother (Tohotoa et al, 2009). 
Rempel et al (2017) identified that fathers' breastfeeding influence behaviour of being 'savvy', termed as 'learning about breastfeeding and using the knowledge to encourage the mother and suggest solutions to breastfeeding problems', was the least demonstrated type of support to the mother's breastfeeding. In contrast to other studies, Rempel et al (2017) concluded that when fathers used their knowledge to support the mother, breastfeeding duration was shorter.

\section{Breastfeeding support from fathers}

Fathers' support was a common theme in the literature. Several papers established that mothers valued fathers' support and this influenced the duration of breastfeeding (Tohotoa et al, 2009; Datta et al, 2012; Nickerson et al, 2012). Nickerson et al (2012) found mothers felt the support of the father was imperative to them continuing breastfeeding. Some studies illustrated the view that mothers believed without the presence and support of the father, they would not have been able to continue when facing breastfeeding challenges (Tohotoa et al, 2009; Datta et al, 2012; Nickerson et al, 2012).

Many mothers identified fathers as 'just being there' to offer support and assistance as an important role for fathers (Tohotoa et al, 2009), especially in the first few weeks after giving birth. This was clear in the study by Nickerson et al (2012), who found that most mothers perceived their husband to be a 'partner' in breastfeeding. This became especially apparent in circumstances where mothers felt they needed additional support from their husband (first-time mothers, mothers recovering from caesarean sections and during breastfeeding challenges).

Partner emotional support in successful breastfeeding was also a theme in the literature. Many of the papers identified that mothers valued this emotional support during the breastfeeding process (Tohotoa et al, 2009; Datta et al, 2012; Nickerson et al, 2012; Mannion et al, 2013; Rempel et al, 2017). Mothers reported that the beginning of breastfeeding was especially difficult (Nickerson et al, 2012). Mothers perceived encouragement, affirmation and reassurance as key factors in fathers providing emotional support (Tohotoa et al, 2009; Nickerson et al, 2012; Mannion et al, 2013). Similarly, fathers identified encouragement as a method used to support the mother to continue breastfeeding (Datta et al, 2012). Both mothers and fathers reported that the father showing kindness and affection was a valued element of emotional support, with fathers assuming a loving and supportive role (Datta et al, 2012).

\section{"Previous research has found that if fathers have a positive attitude and the appropriate skills to support breastfeeding, they can have a significant impact «}

Tohotoa et al (2009) suggested that acknowledgment of the effort in breastfeeding was an aspect mothers found especially important. Mothers (22\%) indicated in one study (Mannion et al, 2013) that their partner felt indifferently or negatively about breastfeeding. Negative comments included breastfeeding being 'too timeconsuming' (Mannion et al, 2013).

Interestingly, Rempel et al (2017) found when fathers wanted the mother to breastfeed for longer, showed appreciation, and were present and informed (savvy) about breastfeeding, mothers actually breastfed for a shorter time.

An equally important factor for mothers was the practical support offered by fathers (Tohotoa et al, 2009; Datta et al, 2012; Nickerson et al, 2012; Mannion et al, 2013). Nickerson et al (2012) found the mother's experience of physical support from the father was a common theme. This included help with household tasks, bringing items to the mother while she was breastfeeding, physically helping the mother to nurse and assisting her in public. In other papers, mothers also reported similar practical ways in which the father supported her (Tohotoa et al, 2009; Mannion et al, 2013). Fathers also felt that doing as much as possible to practically support the mother was something they could do effectively in their role (Datta et al, 2012).

\section{Discussion}

The literature supports the father's role in breastfeeding. In particular, fathers are pivotal supporters when they are recipients of education and information about breastfeeding. Mothers welcome their support, especially with the practical work around the home. They are also a motivator and encourager in the early attempts at breastfeeding.

Previous research has found that if fathers have a positive attitude and the appropriate skills to support breastfeeding, they can have a significant impact (Clifford and McIntyre, 2008). However, a recent study found that interventions are needed to engage fathers more in the breastfeeding decision (Brown and Davies, 2014). Research has also shown that fathers are interested in breastfeeding and want to be involved (Sheriff et 


\section{Key points}

- Research suggests that fathers can be an important source of breastfeeding support but are not acknowledged in many existing breastfeeding protocols or policies

- Fathers are pivotal when they are recipients of education and information about breastfeeding

- Mothers welcome their support, especially with the practical work around the home. They are also a motivator and encourager in the early attempts at breastfeeding

- There is a need for existing policies and guidelines to acknowledge the father's role

- Resources and interventions for fathers need to be designed and researched

al, 2009). However, fathers have previously reported feeling that they lack knowledge and want more information to be proactively involved and able to support their partners (Sheriff et al, 2009). There is not a vast amount of literature on this topic and throughout this article the limitations of the research reviewed has been discussed. Nonetheless, against a backdrop of low breastfeeding rates and the positive impact that breastfeeding has on the health, attachment of child and mother and the bonding of the family, this body of evidence is important (UNICEF, 2013; Gutman et al, 2009; Field, 2010; Wave Trust and Department for Education, 2013; PHE, 2016).

The health visitor working day to day with mothers trying to breastfeed should be aware of the potentially valuable role of fathers in supporting the mother and include them in the advice they give the mothers. Typically, health visitors, while not discouraged during training from engaging with fathers on this topic, are not given information about how to support fathers. In practical terms, there are no leaflets, websites or other sources of information that are 'father friendly'.

Many relevant international and national policies and guidance are not explicit about the positive role fathers could play in supporting mothers. Thus, while there is a strong push for health visitors to support breastfeeding mothers, there is no onus on them to include fathers in their approach. Such an approach could include additional guidance on how to engage with fathers in these circumstances, and the development of resources and toolkits to help health visitors in the community, as well as positive public health communications about the role of fathers as breastfeeding supporters.

\section{Limitations of the study}

This literature review identified only nine relevant studies, which makes it difficult to generalise the findings. In addition, the relevant studies employed different methods, making comparison of findings challenging. However, the wider support network for mothers trying to breastfeed is important and there remains a lack of research in this area. Thus, even with a small evidence base it is important to consider the role of the father.

\section{Conclusion}

As discussed, there is a lack of research in this important area. Breastfeeding researchers and clinical academics should look closely at the father's role, understand how they can be supportive and what resources they need to support them. There is a need to build on the interventions (educational, peer support, father 'friendly' resources) described in this article and to trial them as larger-scale studies with more diverse groups of fathers.

This article has been subject to peer review.

Abbass-Dick J, Stern SB, Nelson LE, Watson W, Dennis CL. Coparenting breastfeeding support and exclusive breastfeeding: a randomized controlled trial. Pediatrics. 2015 Jan 01;135(1):102-110. doi:10.1542/ peds.2014-1416.

American Academy of Pediatrics. Breastfeeding and the Use of Human Milk. Pediatics. 2012;115(3):827-841.

Bartok C), Ventura AK. Mechanisms underlying the association between breastfeeding and obesity. Int J Pediatr Obes. 2009 Jan;4(4):196-204. doi:10.3109/17477160902763309.

Bar-Yam NB, Darby L. Fathers and breastfeeding: a review of the literature. J Hum Lact. 1997 Mar;13(1):45-50. doi:10.1177/089033449701300116.

Braun V, Clarke V. Using thematic analysis in psychology. Qual Res Psychol. 2006 Jan;3(2):77-101. doi:10.1191/1478088706qp063oa. Brown A, Davies R. Fathers' experiences of supporting breastfeeding: challenges for breastfeeding promotion and education. Matern Child Nutr. 2014 Oct;10(4):510-526. doi:10.1111/mcn.12129.

Clifford J, Mclntyre E. Who supports breastfeeding? Breastfeed Rev. 2008 Jul;16(2):9-19.

Critical Appraisal Skills Programme (2014) CASP Checklists. www.casp uk.net/casp-tools-checklists (accessed 1 May 2018)

Datta J, Graham B, Wellings K. The role of fathers in breastfeeding: decision-making and support. Br J Midwifery. 2012 Mar;20(3):159167. doi:10.12968/bjom.2012.20.3.159.

Department of Health (2009) Healthy Child Programme: Pregnancy and the First 5 Years of Life. www.gov.uk/government/publications/ healthy-child-programme-pregnancy-and-the-first-5-years-of-life (accessed 1 May 2018)

Department of Health (2013) Infant Feeding Profiles 2010-2011. www.gov.uk/government/publications/infant-feeding-profiles2010-to-2011 (accessed 1 May 2018)

Department of Health. (2014) Early Years High Impact Area 3 - Breastfeeding (Initiation and Duration). www.gov.uk/government/publications/commissioning-of-public-health-services-for-children (accessed 1 May 2018)

Field F (2010) The Foundation Years: preventing poor children becoming poor adults. http://webarchive.nationalarchives.gov. uk/20110120090128/http:/povertyreview.independent.gov.uk/media/20254/poverty-report.pdf (accessed 1 May 2018)

Gutman LM, Brown J, Akerman R. Nurturing Parenting Capability: The Early Years, Centre for Research on the Wider Benefits of Learning. London: The Institute of Education; 2009.

Horta BL, Victora CG. Long-term effects of breastfeeding: a systematic review. Geneva: World Health Organization; 2013.

Hunter T, Cattelona G. Breastfeeding initiation and duration in first- 
time mothers: exploring the impact of father involvement in the early post-partum period. Health Promot Perspect. 2014 Dec 30;4(2):132136.

Mannion CA, Hobbs AJ, McDonald SW, Tough SC. Maternal perceptions of partner support during breastfeeding. Int Breastfeed J. 2013;8(1):4 doi:10.1186/1746-4358-8-4.

Maycock B, Binns CW, Dhaliwal S, Tohotoa J, Hauck Y, Burns S, Howat P. Education and support for fathers improves breastfeeding rates: a randomized controlled trial. J Hum Lact. 2013 Nov;29(4):484-490. doi:10.1177/0890334413484387.

McAndrew F, Thompson J, Fellows L, Large A, Speed M, Renfrew MJ. (2012) Infant Feeding Survey 2010. https://discover.ukdataservice.ac.uk/ catalogue/?sn=7281\&type=Data\%20catalogue\#publication (accessed 1 May 2018)

McCrory C, Layte R. Breastfeeding and risk of overweight and obesity at nine-years of age. Soc Sci Med. 2012 Jul;75(2):323-330. doi:10.1016/j. socscimed.2012.02.048

National Institute for Health and Care Excellence (2015) Postnatal care up to 8 weeks after birth. www.nice.org.uk/guidance/cg37 (accessed 1 May 2018)

NHS England (2015) NHS England Statistical Release Breastfeeding Initiation \& Breastfeeding Prevalence 6-8 weeks Quarter1 2015/16. www.england.nhs.uk/statistics/wp-content/uploads/sites/2/2014/03/ Breastfeeding-1516Q11.pdf (accessed 1 May 2018)

Nickerson LE, Sykes AC, Fung TT. Mothers' experience of fathers' support for breast-feeding. Public Health Nutr. 2012 Sep;15(09):1780-1787. doi:10.1017/S1368980011003636.

Özlüses E, Çelebioglu A. Educating fathers to improve breastfeeding rates and paternal-infant attachment. Indian Pediatr. 2014 Aug;51(8):654657. doi:10.1007/s13312-014-0471-3.

Public Health England (2016) Breastfeeding at 6 to 8 weeks after birth: 2015 to 2016 quarterly data. Retrieved from the UK government website: https://www.gov.uk/government/statistics/breastfeeding-at-6-to8-weeks-after-birth-2015-to-2016-quarterly-data (accessed 1 May 2018)
Rempel LA, Rempel JK, Moore KCJ. Relationships between types of father breastfeeding support and breastfeeding outcomes. Matern Child Nutr. 2017 Jul;13(3):e12337. doi:10.1111/mcn.12337.

Rempel LA, McCleary L. Effects of the implementation of a breastfeeding best practice guideline in a Canadian public health agency. Res Nurs Health. 2012 Oct;35(5):435-49. doi: 10.1002/nur.21495.

Sherriff N, Hall V, Pickin M. Fathers' perspectives on breastfeeding: ideas for intervention. Br J Midwifery. 2009 Apr;17(4):223-227. doi:10.12968/ bjom.2009.17.4.41670.

Scott JA, Ng SY, Cobiac L. The relationship between breastfeeding and weight status in a national sample of Australian children and adoles cents. BMC Public Health. 2012 Dec;12(1):107. doi:10.1186/1471-2458$12-107$.

Tohotoa J, Maycock B, Hauck YL, Howat P, Burns S, Binns CW. Dads make a difference: an exploratory study of paternal support for breastfeeding in Perth, Western Australia. Int Breastfeed J. 2009;4(1):15. doi:10.1186/1746-4358-4-15.

US Department of Health and Human Services (2011) The Surgeon General's Call to Action to Support Breastfeeding. www.surgeongeneral.gov/ library/calls/breastfeeding/index.html (accessed 1 May 2018) UNICEF (2013) The evidence and rationale for the UNICEF UK Baby Friendly Initiative standards. www.unicef.org.uk/wp-content/uploads/ sites/2/2013/09/baby_friendly_evidence_rationale.pdf (accessed 1 May 2018)

Wave Trust, Department for Education (2013) Conception to age 2 - the age of opportunity. www.wavetrust.org/our-work/publications/reports/ conception-age-2-age-opportunity (accessed 1 May 2018)

World Health Organization (2009) Infant and Young Child Feeding: Model Chapter. www.who.int/nutrition/publications/infantfeeding/9789241597494/en/ (accessed 1 May 2018)

World Cancer Research Fund/American Institute Cancer Research. Policy and Action for Cancer Prevention: Food, Nutrition, and Physical Activity: A Global Perspective. Washington, DC: AICR; 2009.

\section{Call for peer reviewers for Journal of HealthVisiting}

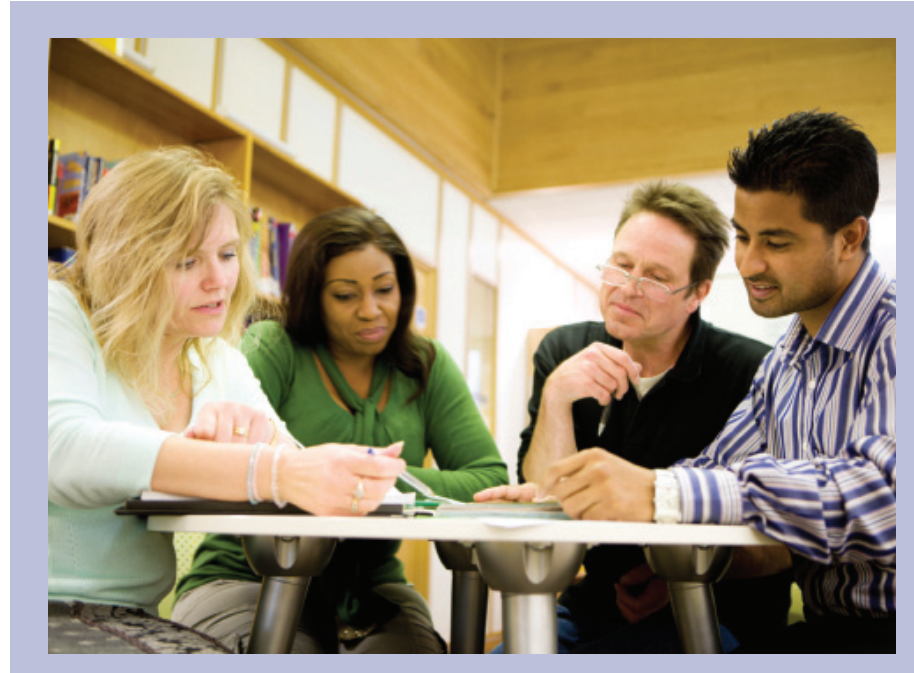

\section{Are you a health visitor, researcher or educator?}

If so, the Journal of Health Visiting would be interested to hear from you as we aim to expand our group of committed peer reviewers.

In reviewing articles, the reviewer is informing and advising the editors, who will make the final decision regarding publication.

If you are interested, please send your CV or contact the editor at jhv@markallengroup.com 